\title{
A Phase 3, Randomized, Open-Label, Multicenter, Global Study of Durvalumab and Bacillus Calmette-Guérin (BCG) Versus BCG Alone in High-Risk, BCG-Naïve Non-Muscle-Invasive Bladder Cancer (NMIBC) Patients (POTOMAC)
}

De Santis M, ${ }^{1}$ Abdrashitov R, ${ }^{2}$ Hegele A, ${ }^{3}$ Parker $S,{ }^{2}$ Palou Redorta J, ${ }^{4}$ Nishiyama $H,{ }^{5}$ Xiao F, Gupta A, ${ }^{2}$ Shore ${ }^{6}$

${ }^{1}$ Charité University Hospital, Berlin, Germany and Department of Urology, Medical University of Vienna, Austria; ${ }^{2}$ AstraZeneca, Gaithersburg, MD; ${ }^{3}$ Department of Urology and Paediatric Urology, Philipps-Universität Marburg, Marburg, Germany; ${ }^{4}$ Department of Urology, Fundació Puigvert, Barcelona, Spain; ${ }^{5}$ Department of Urology, University of Tsukuba, Tsukuba, Japan; ${ }^{6}$ Carolina Urologic Research Center, Myrtle Beach, SC

\section{Introduction}

- Urothelial carcinoma (UC) is the most common type of cancer of the bladder, ureter, urethra, and urachus, accounting for approximately $90 \%$ of primary malignancies of the urinary tract.1,2

- Standard treatment for patients with high-risk non-muscle-invasive tumors is transurethral resection of bladder tumor (TURBT) followed by intravesical administration of BCG. ${ }^{3}$

- Several randomized studies have demonstrated the efficacy of BCG in NMIBC, but recurrence rates are as high as 50\% in the first 3 years of follow-up and can be even higher for aggressive histologic subtypes. ${ }^{4,5}$

- Results of nonclinical and clinical studies of monoclonal antibodies (mAbs) targeting the programmed cell death ligand-1/programmed cell death-1 (PD-L1/PD-1) pathway have shown evidence of clinical activity and a manageable safety profile, supporting the hypothesis that an anti-PD-L1 antibody may therapeutically enhance antitumor immune response in patients with cancer. ${ }^{6-8}$

- Response rates doubling those with traditional chemotherapy have been reported for immunotherapies targeting PD-1 and PD-L1 in trials of patients with metastatic UC who progressed after platinum-based chemotherapy.

- Durvalumab $(D)$ is a selective, high-affinity, engineered human IgG1 mAb that blocks PD-L1 binding to PD-1 and CD80 with a manageable safety and tolerability profile.

- PD-L1 inhibition with $D$ in combination with other immunotherapies, including standard-of-care BCG, may improve response rate and duration of tumor response.

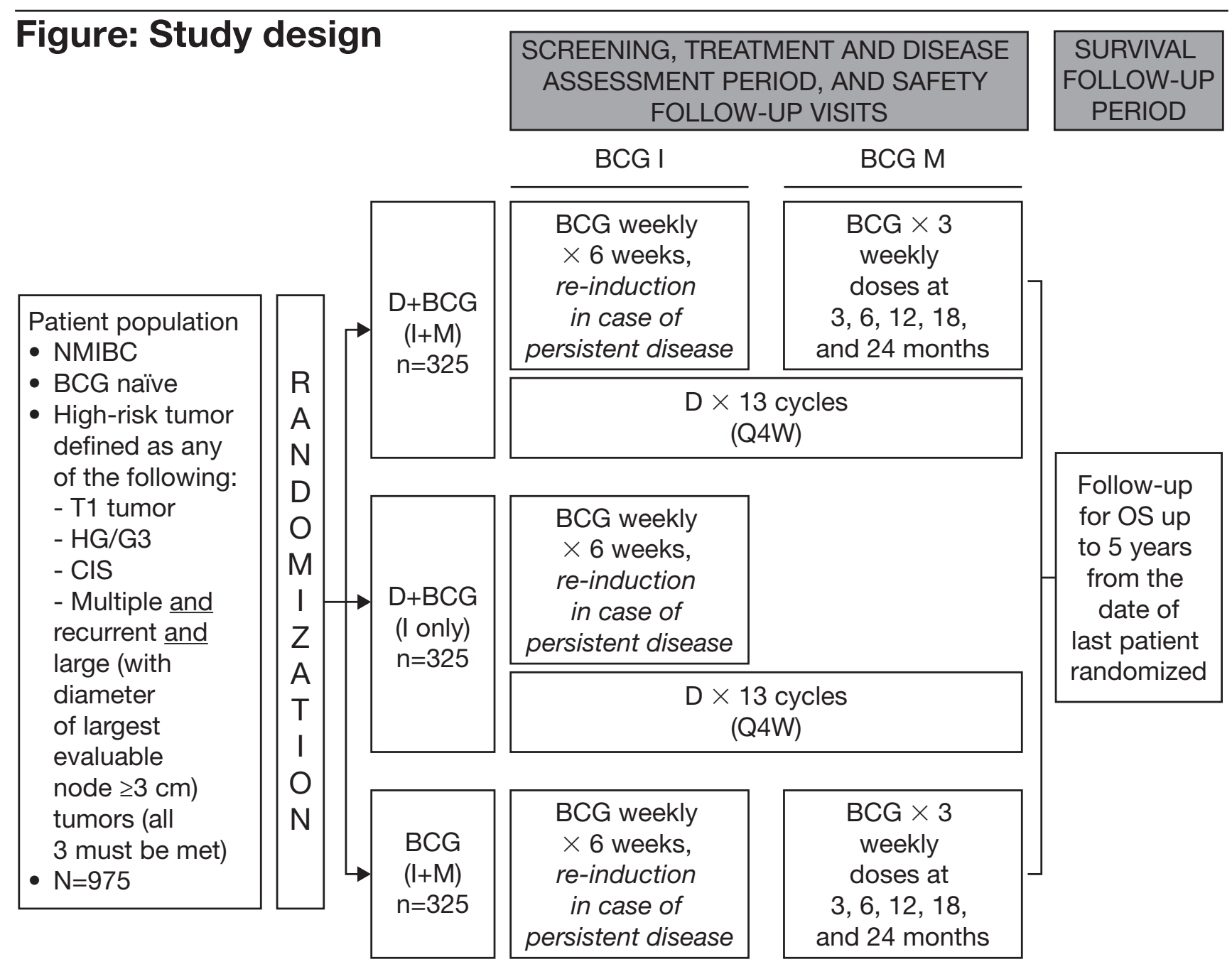

BCG, Bacillus Calmette-Guérin; CIS, carcinoma in situ; D, durvalumab, G, histologic grade; HG, high grade; I, induction; the lamina propria.

\section{Methods}

\section{Study Design}

- This is a randomized, open-label, multicenter, global study to determine the efficacy and safety of $\mathrm{D}+\mathrm{BCG}(\mathrm{I}+\mathrm{M})$ and $\mathrm{D}+\mathrm{BCG}$ (I only) versus $B C G(I+M)$ alone in the treatment of patients with histologically confirmed high-risk NMIBC following complete resection of papillary tumors (patients with residual CIS are eligible) and who are BCG naïve (Figure).

- Approximately 975 patients aged $\geq 18$ years will be randomized (1:1:1) to 1 of 3 treatment arms.

- The primary objective is to assess the efficacy of $D+B C G(I+M)$ compared with $\mathrm{BCG}(\mathrm{I}+\mathrm{M})$ alone as measured by disease-free survival using investigator disease assessments.

- Several secondary and safety objectives will also be explored (Table).

- Treatment will be discontinued if there is high-risk persistent disease or high-risk disease recurrence, disease progression, or unacceptable toxicity.

- Unless discontinuation criteria are met, treatment will continue for $~ 2$ years for the D+BCG $(I+M)$ and BCG $(I+M)$ alone groups and for $~ 1$ year for the $\mathrm{D}+\mathrm{BCG}$ (I only) group.

\section{Table: Additional objectives}

\section{Objectives}

\section{Secondary objectives}

Efficacy of D+BCG (I only) compared with BCG (I+M) alone

Efficacy of D+BCG (I+M) compared with D+BCG (I only)

Disease-related symptoms and health-related quality of life in patients with NMIBC treated with D+BCG (I+M) and D+BCG (I only) compared with $B C G(I+M)$ alone and compared with each

\section{Safety objective}

Safety and tolerability profile of $\mathrm{D}+\mathrm{BCG}(\mathrm{I}+\mathrm{M})$ and $\mathrm{D}+\mathrm{BCG}$ (I only) compared with BCG $(\mathrm{I}+\mathrm{M})$ alone

\section{Study Population: Inclusion and Exclusion Criteria}

\section{Inclusion}

BCG naïve (patients who have not received prior intravesical BCG or who previously received but stopped $B C G>3$ years before study entry)

Local histological confirmation of high-risk transitional cell carcinoma of the urothelium of the urinary bladder confined to the mucosa or submucosa

Complete resection of all $\mathrm{Ta} / \mathrm{T} 1$ papillary disease prior to randomization, with most recent TURBT occurring $\leq 2$ months prior to consent for study

\section{No prior radiotherapy to the bladder}

WHO/ECOG performance status of 0 or 1 at screening

No prior exposure to immunemediated cancer therapy, including, but not limited to, other anti-CTLA-4 anti-PD-1, anti-PD-L1, and anti-programmed cell death ligand-2 antibodies

Adequate organ and marrow function

\section{Conclusions}

- There remains a significant unmet medical need for innovative, effective therapies for NMIBC that can significantly reduce recurrence and progression rates.

- Existing efficacy data and PD-L1 expression data support the potential therapeutic effect of durvalumab in earlier stages of bladder cancer, including high-risk NMIBC.

- PD-L1 inhibition through exposure to durvalumab in combination with the standard-of-care drug BCG, may increase both the long-term response rate and frequency of response by preventing the NMIBC tumor cells from evading immune-mediated antitumor response.

- The addition of durvalumab to BCG treatment may counteract acquired BCG resistance ${ }^{9}$; the combination could provide dual benefit by decreasing recurrence rates and increasing duration of response.

\section{References

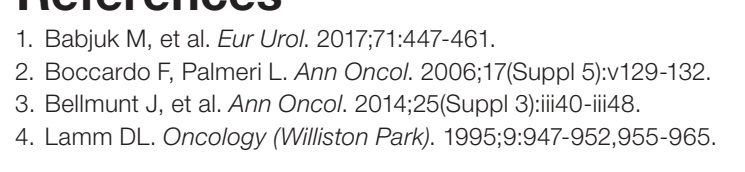

\section{Acknowledgments}

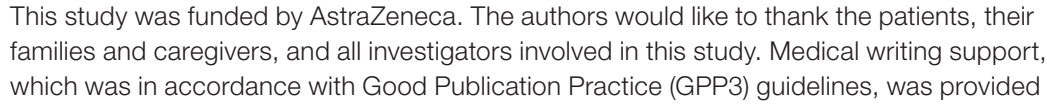

Evidence of muscle-invasive, ocally advanced, metastatic cancer

or documented absolute of adjuvant intravesical BCG

Concurrent extravesical, non-muscle-invasive transitional

Concurrent treatment with systemic immunostimulatory study treatment

Predominantly variant histology (other than urothelial) as assessed by pathology 